\title{
Lo que cuentan las estrellas. El oficio de los astrónomos en la Antigua Mesopotamia
}

\author{
What the stars tell. \\ The astronomer's job in the Ancient Mesopotamia
}

Piedad Yuste*

\begin{abstract}
RESUMEN
Desde épocas muy remotas, los habitantes de la antigua Mesopotamia, habían interpretado los sucesos del cielo como fenómenos premonitorios.

Pequeños grupos de expertos y eruditos, dedicados a observar los astros y sus movimientos, escribieron series de presagios que afectaban al rey y al

Estado. Con el paso del tiempo, la sistematización de estas observaciones desembocó en un verdadero conocimiento científico. En este trabajo vamos a seguir los pasos de los escribas que crearon la

astronomía.
\end{abstract}

ABSTRACT

Since ancient times, the inhabitants of ancient Mesopotamia had interpreted the events of the sky as premonitory phenomena. Small groups of experts and scholars, dedicated to observing the stars and their movements, wrote series of omens affecting the king and the State. Over time, the systematization of these observations led to a real scientific knowledge. In this paper we will follow in the footsteps of the scribes who created the astronomy.

PALABRAS CLAVE:

Astronomía arcaica, Mesopotamia, astronomía babilónica.

KEYWORDS:

Babylonian astronomy, Mesopotamia, early astronomy.

\section{INTRODUCCIÓN}

Para las poblaciones fluviales del Neolítico asentadas en Mesopotamia, cuyo alimento y prosperidad dependía casi exclusivamente de la agricultura, controlar los períodos estacionales podía ser un buen motivo para observar el firmamento.

* Universidad Nacional de Educación a Distancia. UNED. Departamento de Filosofía. Paseo Senda del Rey, no 7, Madrid 28040. E-mail: pyuste@ fsof.uned.es 
También porque creyeron que el cielo era el hogar de los dioses y que éstos utilizaban los astros para comunicarse con la humanidad. Al monarca le convenía demostrar la alianza que mantenía con la divinidad; así, conocer en qué momento debían iniciarse las labores de siembra, calcular la llegada de las lluvias y de los vientos, predecir días más largos y noches más cortas, temporadas cálidas o frías, era tan importante como descifrar el lenguaje de las estrellas. Porque en los cielos se buscaba información sobre la conveniencia de comenzar una batalla o desencadenar una guerra; si peligraba la situación del monarca o la de sus herederos; si vendrían épocas de escasez y hambruna o, por el contrario, se avecinaban tiempos de abundancia y bienestar. Habían comprobado que cada uno de estos sucesos coincidía con un eclipse de Luna o de Sol, o con cualquier otro fenómeno meteorológico relevante: inundaciones, sequías, temblores de tierra, tormentas $^{1}$; y si no era así, inspeccionaban el cielo hasta averiguar qué posición exacta ocupaban los planetas en el momento de producirse determinado acontecimiento en la Tierra.

No se trataba de una relación causa efecto, sino de una señal ${ }^{2}$, un presagio: los dioses avisaban a los humanos de la posible inminencia de ciertos hechos, componiendo frases con las estrellas. Pero sólo unos pocos estaban capacitados para entender la escritura celestial ${ }^{3}$ : Ios llamados țupšar de Enūma Anu Enlil..., los escribas educados para interpretar los mensajes enviados por Anu y Enlil, los dioses del cielo y la tierra ${ }^{4}$, respectivamente:

«Entonces alcé los ojos hacia las tierras bajas,

miré las estrellas al este,

alcé los ojos hacia las tierras altas,

miré las estrellas al oeste,

contemplé el cielo donde se inscribían los signos,

en este cielo inscrito aprendí los presagios,

vi cómo había que aplicar las leyes divinas,

estudié las decisiones de los dioses. $»^{5}$

La sucesión en el tiempo de dos acontecimientos insólitos, tales como un eclipse de Luna y el fallecimiento del monarca, pudieron estar detrás del origen de las series de presagios ${ }^{6}$. Así, a partir de entonces, un eclipse anunciaría desagra-

1 Todos estos sucesos naturales se engloban en la misma categoría de fenómenos meteorológicos.

2 BOTTÉRO, J.: 1974, 150. Acerca de una posible relación causa-efecto de los sucesos estelares, ver LEOUX, D.: 2002.

3 Ver ROCHBERG, F.: 2004, acerca de la «escritura celestial» en las páginas introductorias.

4 Anu y Enlil y Enki (dios de las aguas), son las principales deidades sumerias, según aparecen en el mito cosmológico de Atrahasīs y a ellos están dedicadas estas series de presagios celestiales. Ver LARA PEINADO, F.: 1984, 307.

5 Mito sumerio de «Innana y Šukallituda, o el pecado mortal del jardinero», en KRAMER, S. N.: 2010, 108.

6 Según parece, un eclipse de Luna precedió a la muerte de dos reyes: Maništušu, hijo de Sargón de Acad, y Naram-Sin, nieto de éste. Ver HUBER, P.: 1987 y M. T. LARSEN, M. T.: 1987. También LEHOUX, D.: 2002. 
cias, aunque, como veremos después, también pudo favorecer al rey Sargón de Acad en su batalla contra el monarca Lugalzagesi.

El mundo, an-ki, se concebía finito y circular ${ }^{7}$ : la Tierra plana y quieta, cubierta por una bóveda estrellada, por la que transitan diariamente el Sol, la Luna y los planetas conocidos: Mercurio, Venus, Marte Júpiter y Saturno. A simple vista, parecía muy difícil comprender el comportamiento de los astros: sus movimientos diarios hacia occidente y sus desplazamientos anuales, más lentos, hacia oriente, por la misma ruta. Algunos describían insólitas curvas; avanzando unas veces y retrasándose otras, como hacían Venus y Marte. La Luna viajaba más deprisa que el Sol y había grupos de estrellas que rotaban cada día sin cambiar sus posiciones relativas. El firmamento era pues sorprendente y los humanos lo contemplaron con temor. No obstante, advirtieron cierta rutina en los movimientos del Sol y de la Luna, que les permitió elaborar calendarios, a partir de los cuales fijaron los días festivos dedicados a sus dioses.

Entre los servidores del rey, un grupo de astrónomos y adivinos escrutaban el cielo nocturno buscando indicios y advertencias; asimismo, indagaban en las entrañas de los animales sacrificados y estudiaban la evolución de las gotas de aceite derramadas en el agua. Los escribas dedicados a estos menesteres eran personas instruidas ${ }^{8}$ que registraban cuidadosamente los sucesos observados en la naturaleza e intentaban predecir las consecuencias que de ellos derivaban, componiendo Series de Presagios y Pronósticos, como los celestiales de Enūma Anu Enlil. Hacían partícipes de sus conocimientos únicamente a los miembros de su profesión: a aquellos que saben; herederos estos últimos del sabio Enmeduranki, quien fue aleccionado directamente por los dioses de la adivinación, Šamaš y $\operatorname{Addad}^{\ominus}$. Lo vemos en algunos colofones escritos en los textos:

"Conocimiento exclusivo de los grandes dioses. El que sabe puede mostrarlo a otro que no sabe. El que no sabe no puede verlo. Pertenece a los asuntos prohibidos por los grandes dioses. Escrito de acuerdo a su original y cotejado.»10

Durante siglos, desde el período sumerio hasta el arsácida, y en las cortes de Asiria y Babilonia, estos eruditos desempeñaron su oficio, primeramente, en los palacios, y más adelante, en los templos ${ }^{11}$. El saber se transmitía de padres a hijos, o dentro del entorno familiar; los expertos iniciaban a sus discípulos mediante el juramento:

\footnotetext{
7 Idea expresada en sumerio: «an.ki.nigin.na» y también en lengua acadia: «kippat šame u erȘeti», equivalente a «el círculo del cielo y la tierra», en ROCHBERG, F.: 2004, 127.

8 OPENHEIM, A.L.: 1966, 40.

9 Enmeduranki de Sippar fue el séptimo rey de Sumeria antes del Diluvio. Ver ROCHBERG, F.: 2004, 215.

10 HUNGER, H.: 1968, no 77. F. ROCHBERG, F.: 2004, 212. Ver también ROBSON, E.: 2008, 245 y ss.

11 Tras la caída de Babilonia por Darío II, hacia el año 539, parece que se efectuó este traslado; ver ROCHBERG, F.: 2004, 209.
} 
«El estudioso, el que sabe, el que guarda los secretos de los grandes dioses, se unirá a su hijo, al que ama, con un juramento ante Šamaš y Addad, en presencia de la tablilla y el estilo, y le instruirá.» ${ }^{12}$

La mayoría de los textos que conservamos están escritos en lengua acadia, con signos cuneiformes, sobre tablillas de arcilla. Uno de los documentos más arcaicos que contienen presagios, hace alusión a las escaramuzas emprendidas por el rey Sargón de Acad (2334 a C.), a quien los dioses eran propicios, pues en una de aquéllas «el Sol empezó a oscurecer y las estrellas se hicieron visibles al enemigo » ${ }^{13}$, logrando así la victoria. Su homónimo neoasirio, Sargón II (714 a C.), también venció a las tropas de Urartu gracias a la beneficiosa influencia de un eclipse lunar.

Poco sabemos de los honorarios percibidos por estos expertos y eruditos; el Código de Hammurabi ${ }^{14}$ no contiene ninguna entrada que aluda a esta profesión. Probablemente, además del sustento, los soberanos les obsequiaban con terrenos, plata, enseres y regalos, dependiendo del éxito conseguido ${ }^{15}$ y del favor real; nada seguro y vitalicio, desde luego. Lo vemos en algunos documentos procedentes de los intercambios epistolares entre los reyes de Asiria, Esarhadon y Asurbanipal, y sus consejeros respectivos (siglo VII a C.); también en las cartas encontradas en los Archivos Reales de Mari $^{16}$. En un texto posterior, hallado en el templo dedicado a Marduk, en Babilonia, y fechado en la segunda centuria antes de nuestra era, se alude al estipendio asignado a un escriba de Enūma Anu Enlil que ha sucedido en el puesto a su padre: recibirá un salario anual de una mina ${ }^{17}$ de plata y tierras de cultivo ${ }^{18}$. Sin embargo, se desconocen los vínculos que les relacionan al personal del templo.

De alguna manera, sabemos qué ocupaciones desempeñaban estos eruditos en la corte del rey Asurbanipal:

"Yo soy experto en la profesión de mi padre, la disciplina del lamento; he estudiado y cantado las Series. Estoy versado en lavar la boca y purificar el palacio. He examinado el cuerpo saludable y el enfermo. He leído Enüma Anu Enlil y he realizado observaciones astronómicas. He leído los Šumma izbu, las (obras fisiognómicas)... y las Šumma ālu.»19

Como menciona el escriba, había otras Series de Presagios ${ }^{20}$ : las elaboradas a partir de observaciones directas realizadas en los cuerpos y en el rostro de las

12 Ibid., p. 215.

13 HIRSCH, H.: 1963. REINER, E.: 1999, 24.

14 LARA PEINADO, F.: 1982.

15 OPPENHEIM, A. L.: 1966, 40.

16 PARPOLA, P.: 1970.

17 Mina, ma-na, equivalente a 500 gramos de plata.

18 Ver McEWAN, G. J. P.: 1981, 17 y ss.

19 Este fragmento corresponde a una carta de Marduk-šāpik-zēri, experto en adivinación celestial, al rey Asurbanipal. Ver PARPOLA, S.: 1970, 122 y ROCHBERG, F.: 2004, 5.

20 Ver, por ejemplo, OPPENHEIM, L.: 2003 y ROCHBERG, F.: 2004. 
personas (alamdimmû), que delataban su carácter y comportamiento; listados de diagnósticos y pronósticos médicos (SA.GIG); colecciones de sueños (ziqĩqu) y sus consecuencias; registros de nacimientos anómalos y malformaciones (summa $i z b u$ ), tanto en humanos como en animales; series hemerológicas (iqqur ippuš), que distinguen los días propicios de los que no lo son; y, por último, las series que aluden a la situación y características geográficas (šumma ālu). El fragmento anterior nos hace pensar que el curriculum de un escriba integrado en la corte neoasiria abarcaba todas estas disciplinas. Pero la lengua acadia distingue entre las funciones desempeñadas por el médico exorcista (āšipu), el intérprete de los sueños (šāilu) y el astrólogo (țupšarru). El arúspice (bāru), es el que inspecciona las entrañas del animal en busca de señales premonitorias y advertencias acerca de la conveniencia de emprender alguna acción. Lo cual nos indica que había diferentes técnicas adivinatorias ejercidas por sus correspondientes especialistas. Pero, contrariamente a lo que ocurría con los presagios astrales, que se dirigían al rey y al estado, estas otras clases de pronósticos también podían ser utilizadas por las personas corrientes, siempre que pudieran abonar los honorarios del adivino. Fuera de Enūma Anu Enlil encontramos algunas tablillas dedicadas a los nacimientos:

«(Si) un niño ha nacido y durante su infancia ocurre un eclipse solar: morirá en una ciudad extranjera y la casa de su padre se disipará.» ${ }^{21}$

Posteriormente, y según se desprende de un texto babilonio del período heIenístico, el escriba de Enūma Anu Enlil, asociado al personal del templo, solamente debía realizar observaciones, preparar tablas astronómicas y escribir presagios $^{22}$. Estas tres categorías de textos, empíricos, predictivos y adivinatorios, comenta Francesca Rochberg, formaban parte de la misma tradición ilustrada ${ }^{23}$; las dos primeras caracterizan a la astronomía científica, mientras la última conserva aún las huellas de un largo proceso de creación y transformación. En el contexto que analizamos, no se diferencian de modo explícito la astronomía de la astrología; así pues, hablaremos exclusivamente de astronomía y de astrónomos; de una etapa inicial, adivinatoria y experimental, que observa, anota y predice acontecimientos basándose en registros anteriores. De su evolución hacia una astronomía que explora, calcula y predice; compone tablas numéricas y enuncia reglas y algoritmos con los que anticipar las posiciones y movimientos de los astros, sus primeras y últimas apariciones y otros fenómenos estelares; detalles todos estos exigidos al conocimiento científico.

${ }^{21}$ ROCHBERG, F.: 2004, 62. Los paréntesis indican aclaraciones realizadas por el editor del texto.

22 McEWAN, G. J. P.: 1981, 17 y ss. En las bibliotecas de estos eruditos se han encontrado textos astronómicos, series de presagios, lamentaciones y conjuros, según se desprende de las excavaciones realizadas en las que debieron ser sus viviendas familiares. Ver Robson (2008, pp. 220 y ss.) 


\section{PRESAGIOS ESTELARES}

Los presagios se componen de dos partes: prótasis (antecedente) y apódosis (consecuencia); o lo que es lo mismo: «Si observamos tal fenómeno», entonces «sucederá determinada cosa». Mientras la prótasis describe un suceso astronómico (o de cualquier otra clase), en la apódosis se pronostica acerca de la persona del rey o su familia, el estado y sus enemigos; también se anuncian desgracias y bonanzas para el país. Aunque se contemplaba la posibilidad de que estos sucesos fueran neutralizados mediante ritos apotropaicos y hechizos (namburbi). El exorcista recurría a plegarias, brebajes y amuletos para detener el acontecimiento vaticinado. Tratándose de presagios estelares, en la mayoría de los casos la apódosis anunciada no se cumpliría; así, la práctica de esos ritos habría de considerarse muy útil y necesaria.

Se han encontrado series de eclipses lunares, correspondientes al período paleobabilónico (2000 a 1600 a C.), que no se ajustan a este esquema y que, probablemente, precedieran a otras series ${ }^{24}$ :

«Un eclipse observado por la tarde, anuncia plagas».

«Un eclipse observado a mediodía, anuncia debilitamiento de la economía».

«Un eclipse en su zona media; se oscurece todo y después se aclara todo: el rey morirá; destrucción de Elam.»

Las zonas de la Luna, ensombrecidas por la Tierra, incumben dramáticamente a las regiones geográficas de Mesopotamia, a las que presagia su destrucción:

«Un eclipse en occidente: la ruina de los amoritas.»

«Un eclipse en el norte: la ruina de los acadios.»

«Un eclipse en oriente: la ruina de Subartu.»

«Una Luna surge misteriosamente y se aclara: pronóstico de destrucción de Elam y Gutea.»

La serie Enūma Anu Enlil, o Cuando Anu y Enlil..., según reza el incipit de la primera tablilla, se encontró en la Gran Biblioteca que el rey asirio Asurbanipal mandó levantar en Nínive, allá por el siglo VIII a. C. Pero su origen parece ser anterior $^{25}$. Esta serie consta de 70 tablillas de arcilla escritas en lengua acadia y en ellas se registran los presagios derivados de las observaciones realizadas al Sol, la Luna, planetas conocidos ${ }^{26}$, fenómenos meteorológicos y estrellas; también, los eventos asociados (apódosis) a esas circunstancias: subidas de precios, estado de

${ }^{23}$ ROCHBERG, F.: 2004, 55. Por el contrario, E.REINER opina lo contrario (1999, p. 31).

24 Se trata de una tablilla depositada en el Museo Británico: BM 22696 (Ibid., p. 69).

25 Ver HUNGER, H. \& PINGREE, D.: 1999, 12 y ss.

${ }_{26}$ Planetas errantes o «intérpretes» de la voluntad de los dioses. Sus ortos y ocasos anuncian terremotos, tormentas, lluvias, vientos, calor, eclipses, etc. Sucesos buenos y malos. 
las cosechas, nivel de las aguas, etc. Se hicieron numerosas copias de este documento y se trasladaron a Hattusa (hoy día Bogazköy), capital del imperio hitita, y a Ugarit.

Las primeras 22 tablillas recogen presagios relacionados con Sin, el dios Luna. Se anota la forma de sus cuernos, el halo que la rodea, sus primeras y últimas apariciones; el color, sombra y dirección del eclipse, cuándo se produce. Cada uno de estos fenómenos es señal de un auspicio. Para los babilonios fue decisivo, además, predecir el momento en que la Luna se hacía visible por primera vez después de la conjunción con el Sol (Luna Nueva). Esto marcaba el inicio del mes y decidía el comienzo del año cuando este fenómeno coincidía, más o menos, con el equinoccio de primavera ${ }^{27}$. La duración del día, entonces, era la misma que la noche. Se medían estos tiempos con clepsidras o relojes de agua, calculando el peso, en minas, del agua desalojada.

Nuestro satélite se desplaza por el firmamento cambiando progresivamente su forma y brillo, hasta alcanzar su plenitud en la fase de oposición con el Sol (Luna Llena). Los eclipses sucederían, precisamente, en estos puntos de alineación solar; sin embargo la inclinación de la trayectoria de la Luna respecto de la del Sol (5ํ), disminuye esta posibilidad.

Las tablillas 23 a 36 enuncian pronósticos relativos al dios Sol, Šamaš. Se examinan sus movimientos, corona, posiciones en relación a las estrellas (MUL) eclípticas $^{28}$, eclipses, etc. Las 13 siguientes registran presagios relacionados con los fenómenos meteorológicos: tormentas, relámpagos, truenos, terremotos, arco iris, formaciones nubosas, vientos, lluvias y tempestades; por tanto, están dedicadas al dios Adad. Las últimas tablillas, de la 50 a la 70, se refieren a la diosa Ištar y anotan los ortos helíacos ${ }^{29}$ de los planetas, posiciones relativas, fases, primeras y últimas visibilidades, luminosidad, color, etc. Todos ellos en cuanto anuncian sucesos favorables o adversos:

«Si al salir Venus, la estrella Roja entra en él: el hijo del rey tomará el trono.»

«Si el Arco se aproxima a UD.AL.TAR (Júpiter): Elam comerá buenos alimentos. ${ }^{30}$

La tablilla $\mathrm{n}^{\circ} 63$ de esta colección, popularmente conocida como Tablilla de Venus del rey Ammisaduqa (1646-1626 a. C.), recoge datos astronómicos acaecidos durante los 21 años de su reinado. Realiza un seguimiento exhaustivo de la trayectoria del planeta, anotando las fechas y sus consecuencias:

27 Uno de los puntos de corte entre las imaginarias circunferencias ecuatorial y eclíptica; ésta última es la línea por la que se desplaza el Sol en su movimiento aparente hacia el este. El otro punto señala el equinoccio de otoño. La eclíptica es la línea donde se producen los eclipses. Las observaciones astronómicas iban dirigidas, principalmente, a predecir este día en años sucesivos.

28 También denominadas estrellas zodiacales, porque discurren por la misma trayectoria que el Sol y pueden contemplarse en el cielo nocturno.

${ }^{29}$ Se llama orto helíaco a la aparición de un astro antes de la salida del Sol.

30 REINER, E. and PINGREE, D.: 1981, 49. 
«Si en el segundo (día) de nisannu ${ }^{31}$ Venus aparece en el este: habrá luto en la tierra; continúa presente en el este hasta el sexto (día) de kis/imu²; desaparece el séptimo de kis/imu y se hace invisible durante tres meses; el octavo (día) de ad$d a r u^{33}$ Venus sale en el oeste: el rey enviará mensajes de hostilidad al rey.» ${ }^{34}$

Del período neoasirio procede el catálogo de estrellas MUL.APIN (constelación del Triángulo), cuya copia más antigua está fechada en el año 687 a. C., pero data, posiblemente, del año 1000 a. C. En él aparecen registradas 60 constelaciones y los ortos helíacos de muchas estrellas: eclípticas y cenitales (ziqpu); además, se anotan los períodos de visibilidad de los planetas, el Sol y la Luna:

«El (día) 15 de nissanu, al comienzo de la noche, la Luna (Sin) está en Libra (rin), en el este, y el Sol (Šamaš) está delante de la estrella posterior de Aries (Iu), en occidente. Tres minas es la duración de la luz y tres minas es la duración de la noche. ${ }^{35}$

Las estrellas y constelaciones transitan diariamente por los Caminos de Anu, Enlil y Ea, según lo hagan a ras del horizonte, o por encima o debajo de éste. Después de las observaciones, el escriba incluye los presagios relativos al bienestar de la corona y del pueblo. La adivinación celestial llegó a Occidente a través de las rutas comerciales establecidas entre Asiria y los pueblos de Anatolia y Siria. También, impregnó las culturas de Egipo y Grecia, durante los períodos persa y helenístico. Posteriormente, alcanzó al mundo romano ${ }^{36}$.

El universo contemplado a simple vista no parecía demasiado grande: grupos de estrellas que giran diariamente; el Sol, que asoma por oriente y se pone por occidente; la Luna, repitiendo sus fases. Convinieron en que el Sol viajaba hacia el este a un ritmo de 1 unidad o grado al día ${ }^{37}$. La Luna lo hacía más deprisa: 13 unidades diarias ${ }^{38}$. Los planetas avanzaban de tal modo que sus velocidades disminuían en función de su lejanía. Sus posiciones se calculaban con referencia a una o varias de las estrellas que circulan por el camino de la Luna. Se conocían hasta 34 de esas estrellas, llamadas Dioses del Consejo, muchas de las cuales se agrupan formando asterismos: son las constelaciones (lumāšu). El método más primitivo de calcular las posiciones de los astros consistía en hacerlo cuando estos pa-

${ }_{31}$ Nisannu era el primer mes del año, que corresponde a nuestro marzo/abril; recordemos que el inicio del mes coincide con nuestro día 15.

32 Kislimmu es el noveno mes, correspondiente a nuestro noviembre/diciembre.

${ }^{33}$ Mes doce; corresponde a febrero/marzo.

34 E. Reiner and PINGREE, D.: 1978, 39.

35 Las cifras deberían ser ordinales, tal como aparecen en los textos.

36 ROCHBERG, F.: 1999, 23.

37 En este contexto, el uš es la unidad del tiempo. En los equinoccios, la duración del día=duración de la noche $=3,0$ uš (12 h.) En el solsticio de verano, la longitud del día = 4,0 uš (16 h); en el solsticio de invierno, el día comprende 2,0 uš ( $8 \mathrm{~h})$. Escribimos las cantidades en base sexagesimal:

$$
a, b, c ; m, n_{(60}=a \times 60^{2}+c+m \times 60^{-1}+n \times 60^{-2}{ }_{(10} \text {. }
$$

A un año de 360 días le corresponderían 36,0,0 uš.

${ }_{38}$ Esta diferencia entre las aparentes velocidades de la Luna y el Sol, provoca que la Luna retrase diariamente su aparición 50 minutos. 
saban junto a una de esas constelaciones, llamadas zodiacales porque se identificaron con un imaginario zoomorfo. Al principio, computaron 18 grupos estelares; mucho después, hacia el siglo $\mathrm{V}$ a $\mathrm{C}$., hicieron corresponder la revolución de los planetas, del Sol y de la Luna con 12 constelaciones zodiacales. Y poco antes de finalizar este mismo siglo, sustituyeron las constelaciones por doce signos zodiacales de $30^{\circ}$ cada uno ${ }^{39}$. Dividieron así la circunferencia eclíptica en 12 partes, coincidiendo con los 12 meses lunares que contiene el año solar. Pero esta metodología no corresponde aún al período que estamos examinando.

En Enūma Anu Enlil podemos leer un ejemplo de cómo se tomaban las referencias respecto de las distintas partes de las constelaciones zodiacales:

«Si Júpiter (SAG.ME.GAR) alcanza la cabeza del Escorpión (GíR.TAB), en la tierra de Acad el mercado (los negocios) se reducirá a la mitad.»

«Si Júpiter alcanza la garganta del Escorpión, en la tierra de Elam el mercado se reducirá a la mitad.»40

El día comienza al ponerse el Sol y corresponde a una rotación completa de los cielos: de puesta a puesta del Sol. Su duración es de 12 danna, o 12 horas dobles ( 1 danna = 30 uš). Es decir, el Sol se desplaza un grado cada 4 minutos.

El año solar se podía computar de dos maneras ${ }^{41}$ :

- Dos pasos sucesivos por el mismo asterismo (nosotros lo llamamos año sidéreo)

- Dos equinoccios vernales consecutivos (denominado actualmente año trópico)

En total, poco más de 365 días al año, con meses de 29 y 30 días $^{42}$. Los babilonios no distinguieron entre año sidéreo y año trópico ${ }^{43}$, diferencia ocasionada por el movimiento de precesión de los equinoccios ${ }^{44}$.

El año lunar comenzaba con la primera aparición en fase creciente de la Luna (después de la conjunción), al pasar junto a las Pléyades o MUL.MUL; fenómeno que coincidía, aproximadamente, con el equinoccio de primavera. Para elaborar el calendario lunar, se computaba el mes sinódico, en el que la Luna repite su fase, y

39 BRACK-BERNSEN, L. \& HUNGER, H.: 1999, 280.

40 REINER, E.: 1999, 26.

41 El año trópico solar consta de 365'2422 días solares; se calcula midiendo el tiempo transcurrido entre dos equinoccios de primavera consecutivos; en los equinoccios de primavera y otoño la duración del día es idéntica a la de la noche. El año sidéreo se calcula midiendo el intervalo temporal entre dos pasos sucesivos por el mismo asterismo; su duración es de 365'2564 días solares medios. Los babilonios no hicieron estos cálculos.

42 Meses con 29 días: aquellos en los que la Luna se hacía visible por primera vez antes de comenzar el día 30. Meses con 30 días: la Luna no era visible tras el ocaso del día 30. Ver BRACK-BERNSEN, L.: 2002, 6.

43 AABOE, A.: 2002, 1.

44 Fenómeno descubierto por Hiparco de Alejandría (ca. 140 a C.). El eje terrestre describe un movimiento circular por el cual los equinoccios se desplazan. 
que dura algo menos de 30 días solares. El dios Marduk organiza los cielos en el Poema Enūma Elišt5:

12. Hizo brillar a Nanna (dios Luna), a quien confió la noche,

13. y le asignó ser la joya nocturna para determinar los días:

14. «Cada mes, sin cesar, ponte en marcha con tu disco;

15. al principio del mes ilumina sobre la tierra,

16. luego guarda tus cuernos brillantes para determinar los seis primeros días;

17. al día séptimo tu disco deberá estar en la mitad,

18. en el decimoquinto te pondrás en conjunción con Šamaš, cada medio mes,

19. y cuando Šamaš, desde la base del cielo, se dirija a ti,

20. con regularidad disminuye (tu disco) y decrece.

21. En el día del oscurecimiento aproxímate a la ruta de Šamaš,

22. para que el [tri]gésimo te encuentres (de nuevo) en conjunción con él».

23. [...] y siguiendo este camino, [define] los presagios;

24. aproxímate $[\ldots]$ para pronunciar las sentencias-adivinatorias.

25. Que Šamaš [...] muertes y expolios.

La Luna atravesaba 18/17 constelaciones a lo largo del año y éste se dividía en doce meses sinódicos ${ }^{46}$. Por tanto, el año lunar constaba de 360 días sinódicos. Así se refleja en Enūma Anu Enlil, MUL.APIN y en algunos astrolabios y catálogos de estrellas correspondientes al primer milenio antes de nuestra era ${ }^{47}$. Quizá este sea el motivo de hacer la circunferencia equivalente a 360 grados y el origen del establecimiento y sistematización del sistema sexagesimal en las matemáticas de los babilonios y asirios. No obstante, existía un desajuste entre ambos calendarios, solar y lunar, que se solventaba añadiendo cinco días en meses alternos.

Con el paso del tiempo, se fueron afinando estos cómputos ${ }^{48}$. El año lunar constaba realmente de poco más de 354 días sinódicos; había, por tanto, un desfase de 11 días respecto al año solar que debían completar intercalándolos hasta hacer coincidir el paso de la Luna por las Pléyades con el año nuevo. Después, añadieron un mes cada tres años. A partir del siglo IV, aproximadamente, introdujeron siete meses extra cada 19 años, pues habían observado la coincidencia entre 19 años tropicales y 235 meses sinódicos. Así, este período contendría un año con un segundo sexto mes $\left(\mathrm{VI}_{2}\right)$ y otros seis años con un segundo duodécimo mes $\left(\mathrm{XII}_{2}\right)^{49}$ a uno de ellos. Metón de Atenas identificó este ciclo en el año 432 a C.; por eso se llama Ciclo Metónico.

45 Tablilla V, ver LARA PEINADO, F.: 1994, 71.

46 Debemos distinguir el mes sinódico (con 29,53 días), en el que se repite una fase, del mes sidéreo, en el cual se determina el tiempo transcurrido entre dos pasos sucesivos por el mismo asterismo. La Luna posee un ciclo sidéreo de poco más de 27 días.

47 HUNGER, H. \& PINGREE, D.: 1999, 50.

${ }^{48}$ El sistema sexagesimal permite realizar cálculos con números fraccionarios con bastante aproximación.

${ }^{49}$ GRAY, J. M. K. \& J. M. STEELE, J. M.: 2008, 556. 
A pesar de estos arreglos, la complejidad de los cielos no parecía aclararse. La perspectiva geocéntrica mostraba un universo en el que los cuerpos celestes poseen movimientos contrarios: hacia delante y hacia atrás (movimiento retrógrado). Algo inexplicable. Actualmente, y gracias al modelo cosmológico propuesto por Kepler, podemos entender el funcionamiento de nuestro sistema solar, aunque nuestros ojos nos engañen.

\section{TRATADOS CIENTÍFICOS}

La astronomía científica surge de manera explícita en el período neoasirio (hacia el año 775 a C.) y abarca los períodos caldeo (neobabilónico), seleúcida y arsácida $^{50}$. Durante estos siglos, se realizaron numerosas y detalladas observaciones del movimiento y posición relativa de los planetas y la Luna, así como de sus alineaciones (conjunciones y oposiciones) respecto del Sol ${ }^{51}$ y otros fenómenos estelares, como datación de los solsticios y equinoccios. Sin embargo, desconocemos a partir de qué astro o astros hicieron sus cómputos y qué métodos e instrumentos utilizaban ${ }^{52}$. En los casos en que el planeta no era visible, se anotaba su posición indicando que las nubes habían impedido observarlo. Hubo muchos cambios en esta época. La confirmación de que los movimientos planetarios se ajustaban a ciclos computables, hasta el punto que era posible anticipar sus posiciones y fases, permitió a los astrónomos desarrollar un complejo sistema algorítmico que desembocó en lo que hoy denominamos conocimiento científico ${ }^{53}$. Los nuevos textos astronómicos no contienen presagios ni alusiones a los dioses, sino tablas, procedimientos aritméticos y predicciones. La rotación de los astros no se atribuye al capricho de los dioses ni los fenómenos del cielo suceden de modo arbitrario o aleatorio. Existe cierta regularidad que puede y debe ser estudiada.

En esta época aparecieron los primeros horóscopos individuales, se consultaban las Series y se recitaban nuevos presagios:

«En el (día) 13 del mes ulūlu (VI), Sin empezó a eclipsar y se puso mientras eclipsaba. Esto es una señal de que el dios Luna solicita una sacerdotisa.»54

Lo cual prueba la vitalidad de esta tradición milenaria, reconocida por el mismo Cicerón ${ }^{55}$. Aunque a partir del siglo II a C., los monarcas ya no requirieron los ser-

50 Casi todos estos textos proceden de Babilonia y Uruk. El último tratado astronómico escrito en lengua acadia data del año $75 \mathrm{~d}$ C.

51 Se medían los arcos sinódicos, es decir, la distancia angular recorrida por el planeta entre dos fases consecutivas de la misma clase: primeras visibilidades, últimas visibilidades, estaciones, etc.

52 BRACK-BERNSEN, L. \& H. HUNGER, H.:1999, 281 y ss. proponen una interesante hipótesis a partir de dos textos cuneiformes que sugieren el uso de un reloj solar. Pero ambos son del período paleobabilónico.

53 MCT: Textos astronómicos cuneiformes, edición a cargo de NEUGEBAUER, O.: 1983; 1ª edición en 1953

54 Presagio estelar fechado en septiembre del año 554; hace alusión al rey Nabónido, último monarca neobabilónico, quien, tras escuchar este presagio, ordenó a su hija hacerse sacerdotisa.

55 Sobre la adivinación (1999). 
vicios de los escribas de Enūma Anu Enlil, adscritos al templo Esagila de Babilonia, pues la capital del reino se había trasladado a Seleucia del Tigris.

Conservamos tres clases de textos astronómicos correspondientes a este período y escritos en lengua acadia:

- Matemáticos: contienen tablas, cálculos, reglas y procedimientos con los que se determinan las posiciones relativas de los planetas y de la Luna. Aquí se aplican dos algoritmos básicos llamados por los historiadores sistemas $A$ y $B$, y que aluden a una hipotética trayectoria descrita por los astros: escalonada o en zigzag ${ }^{56}$. De esta clase son las Efemérides.

- Empíricos: Con observaciones y predicciones, como los Diarios. O solamente predictivos: Ios Almanaques y Almanaques de estrellas eclípticas ${ }^{57}$.

- Horóscopos: Ni observaciones ni predicciones ${ }^{58}$; los datos se tomaron, probablemente, de los Diarios y Almanaques. Se encuadran dentro de la tradición adivinatoria.

Hay también otra clase de tratados que anotan observaciones y predicen los datos correspondientes a los años en los que se cierra un ciclo planetario ${ }^{59}$. Abraham Sachs los llamó Goal-Texts ${ }^{60}$. Los astrónomos asirios y babilonios sabían que Mercurio completaba su ciclo en 46 años; Venus lo hacía en 8; Marte en 47 y 79 años; Júpiter en 83 y 71; Saturno en 59 y la Luna en 19 años. Además, comprobaron que nuestro satélite repite la secuencia de eclipses cada 18 años solares, 0 sea, cada 223 meses sinódicos; estos son los ciclos saros, con los que Beroso, célebre astrónomo al servicio del dios Marduk, computaba el tiempo en su Historia de Babilonia ${ }^{61}$.

Leamos, por ejemplo, varias líneas de un Goal-Year de Júpiter, fechado en el año 140 de la era seleúcida ${ }^{62}$ :

1. [Año 69, reinado de Seleúco]. (Mes) II (día) 3, primera aparición de Júpiter en Aries; era pequeño, salida de Júpiter al salir el Sol: $12^{\circ} 30^{\prime}$.

2. [...] el punto estacionario. (Mes) VIII (día) 3 , salida opuesta de Júpiter. (Mes) X hasta el (día) primero, [punto estacionario de] Júpiter [al oeste]

56 Ver NEUGEBAUER, O.: 1983, I.

57 En inglés, se llaman «Normal Stars».

58 ROCHBERG, F.: 1999 y 2004.

59 Los ciclos indican períodos de tiempo tras los cuales se repiten los mismos fenómenos. Se computaban los períodos sidéreo (tiempo en el que el planeta, aparentemente, da una vuelta completa alrededor de la Tierra) y sinódico (ciclo en el que el planeta repite su fase).

60 A. Sachs (1948, pp. 282-285).

61 «Para realizar los recuentos, Beroso utilizó saroi, neroi y sossoi. El saros es una unidad de tiempo que consiste de 3.600 años, un neros, de 600 años y un sosos de 60 años». Ver Syncellus, Ecloga Chronographica 53, 30, 71-72, en G. WERBRUGGHE, P. \& VICKERSHAM, J. M.: 1999, 47.

62 Se trata de LBAT 1251+1252, en H. HUNGER, H.: 1999, 88-89. Las palabras entre corchetes indican que se han perdido; los paréntesis introducen aclaraciones del editor. 
3. [Año 56,] mes VI intercalado. Año 57, reinado de Antíoco. (Mes) III, noche del (día) 15, última parte de la noche, Júpiter [...]

4. [...] mientras vuelve al oeste, estaba 2 y $2 / 3$ de kùš debajo de Tauro; información oral. (Mes) XI, noche del $21[\ldots]$

Etc.

(Los días y horas se expresan mediante sus correspondientes ordinales: primero, segundo,...)

El kùš (codo) es la medida estándar de longitud; en astronomía, significa distancia angular; sus respectivos submúltiplos se llaman šu.si (dedos); en los documentos procedentes del período paleobabilónico, 1 kùš equivale a 30 šu.si (y a $\left.2,5^{\circ}\right)$; posteriormente, encontramos que 1 kùš equivale a 30 šu.si $\left(2^{\circ}\right)^{63}$. En los Diarios, en cambio, no eran tan explícitos; únicamente apuntaban que, en cierta fecha, el cuerpo celeste $A$ estaba situado -arriba, abajo, delante o detrás- del astro $B$, a una distancia $n$ kùš. Esto indica que computaban la longitud y la latitud de los astros. Leamos un ejemplo fechado en el año 331 a C.:

«Noche del (día) 20, última parte de la noche, la Luna estaba [...kù]š debajo de $\beta$ Géminis, la Luna vuelve 2/3 de kùš hacia el oeste. (Día) 21: el equinoccio; yo no lo vi. La no[che del (día) 22, última parte de la noche,] [la Luna estaba] 6 kùš [debajo de] $\varepsilon$ Leo, la Luna ha pasado $1 \frac{1}{2}$ kùš detrás de $\alpha$ Leo. Noche del (día) 24 , nubes en el cielo. ${ }^{64}$

Las observaciones eran diarias, aunque se anotaban los fenómenos más relevantes acaecidos durante seis o siete meses. El más antiguo de estos tratados está fechado en el año 652 a C., pero hay indicios de que esta práctica comenzó durante el reinado del rey Nabonasar, entre los años 747 y 734 a C. La última copia de un Diario acadio data del año 50 a C. ${ }^{65}$

Pero el asunto que más preocupaba a los astrónomos babilonios y al que dedicaron más esfuerzo ${ }^{66}$, era entender el irregular comportamiento de la Luna: computar sus primeras y últimas visibilidades antes y después de la conjunción y de la oposición; medir la duración de la luz solar en relación a la noche. Por esa razón, introdujeron en sus estudios conjuntos de datos, como los Lunar 3 y Lunar 6 , en los que se registraba el tiempo transcurrido entre los respectivos ortos y ocasos del Sol y de la Luna ${ }^{67}$. El propósito de este estudio iba dirigido, como sabemos, a predecir el inicio del año e intentar ajustar los calendarios solar y lunar.

63 GRAßHOFF, G.: 1999, 112. Ignoramos por qué realizaron este cambio.

64 ROCHBERG, F.: 2004, 8). No estamos seguros a la hora de identificar esas estrellas.

65 Ibid., p. 146.

${ }^{66}$ La mayoría de los documentos que se han podido recuperar están dedicados a estudiar las fases y movimientos de nuestro satélite. Ver NEUGEBAUER, O.: 1983.

67 Además de calcular los tiempos transcurridos entre estos sucesos, se medían los arcos sinódicos o longitudes recorridas entre dos fenómenos consecutivos de la misma clase. Ver BRACK-BERNSEN, L.: 2002, 5-19. 
En relación a los planetas, se anotaban los siguientes fenómenos que, en la actualidad y gracias a Otto Neugebauer, identificamos con letras griegas:

- Para los exteriores, Marte, Júpiter y Saturno:

- Aparición en oriente ( )

- Punto estacionario en oriente ( )

- Oposición ( )

- Punto estacionario en occidente ( )

- Desaparición en occidente ( )

- Los planetas inferiores, Mercurio y Venus, añaden otros dos datos:

- Desaparición en oriente $(\Sigma)$

- Aparición en occidente ( )

Los Almanaques de estrellas y Almanaques, recogían, respectivamente, las posiciones mensuales de las estrellas y de los planetas, en relación a los signos zodiacales. Solían abarcar un año completo, ya fuera de 12 o 13 meses. Contenían datos Lunar 3, Lunar 6 y predicciones. Veamos, por ejemplo, un fragmento extraído de un Almanaque fechado en el año 236 de la era seleúcida (75 d. C.) ${ }^{68}$ :

«Mes III, el primer (día) que sigue al (día) 30 del mes anterior ${ }^{69}$. Júpiter en Géminis, Venus en Cáncer, Saturno en Virgo, Marte en Aries. Hacia el (día) 9 Marte alcanzará Taurus; el (día) 15 la Luna se pone después de la salida del Sol; el (día) 16 el solsticio; hacia el (día) 15 Mercurio será visible por primera vez en el oeste en Cáncer; el (día) 21 Venus alcanzará Leo; el (día) 22 Mercurio alcanzará Leo; el (día) 27, última visibilidad de la Luna antes de la salida del Sol; la noche del (día) 29, habrá eclipse del Sol.»

En las Efemérides se indicaban las posiciones, fases y movimientos de los cinco planetas conocidos y de la Luna. Los cómputos y predicciones, distribuidos en filas y columnas, se realizaban siguiendo dos metodologías diferentes que los historiadores las han identificado con los nombres $A$ (sistema escalonado) y $B$ (sistema en zigzag):

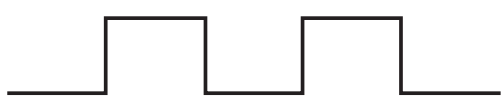

Sistema A

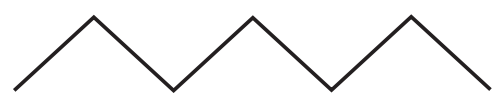

Sistema $B$

No hay duda de que estos tratados tabulares entran ya en el ámbito de la astronomía científica: registro de datos, para las tablas; enunciado de hipótesis, in-

68 Se trata de la tablilla LBAT 1174. Ver ROCHBERG, F.: ibid., 154.

69 Esto significa que el mes anterior se componía de 30 días. Este es uno de los datos que recogen los Lunar 3. Otros meses constaban de 29 días. Ver BRACK-BERNSEN, L: 2002. 
troducción de reglas, aplicación de algoritmos, para los procedimientos ${ }^{70}$. Aunque, de ningún modo, aparecen gráficos como los dibujados más arriba. Nos falta comprobar si los autores de estos textos elaboraron un modelo teórico a partir del cual interpretar los fenómenos observados, como hicieron los astrónomos griegos. Eudoxo y Aristóteles postularon un universo ordenado y finito, formado de esferas concéntricas por las que discurrían los cuerpos celestes, desplazándose con movimiento uniforme en torno a una Tierra inmóvil. Pero la ausencia de pruebas documentales no invalida la posibilidad de que, los astrónomos babilonios hubieran concebido un modelo cosmológico similar. De hecho, los datos numéricos recogidos en las tablas se ordenan, para la función zigzag, en progresiones aritméticas de las que se extraen los valores máximos y mínimos, la amplitud, diferencia, valor medio, número de términos que intervienen en el cálculo y número total de períodos. Asimismo, se establece (como hipótesis) que los cuerpos celestes circulan por el cosmos incrementando o disminuyendo sus respectivas velocidades; y se introducen, además, dos reglas para determinar las posiciones, tiempos y velocidades de los mismos. Así lo vemos en algunos de los procedimientos incluidos en estos tratados ${ }^{71}$. Esto supone un importantísimo avance con respecto a los textos paleobabilónicos Enūma Anu Enlil y MUL.APIN, por ejemplo, en los que las velocidades del Sol y de la Luna se entendían constantes. Las teorías lunar y solar descritas en las Efemérides, según el sistema escalonado, establecen dos velocidades anuales para ambos astros y dos intervalos por los que discurren con cada una de ellas:

1. Desde $13^{\circ}$ Virgo hasta $27^{\circ}$ Piscis, la velocidad del Sol, $V$, es de $30^{\circ}$ cada mes sinódico.

2. Entre $27^{\circ}$ Piscis y $13^{\circ}$ Virgo, el Sol viaja con $v=28^{\circ} 7^{\prime} 30^{\prime \prime}$ cada mes sinódico.

3. Además, se introducen dos factores mediante los cuales se calculan las longitudes recorridas por los planetas en relación al Sol:

- $V / v=1 ; 4$

- $v / V=0 ; 56,15$

La astronomía actual toma como punto de referencia $\left(0^{\circ}\right)$ el equinoccio vernal, situado en Aries; sin embargo, en las Efemérides encontramos este punto desplazado $10^{\circ}$ para el sistema A y $8^{\circ}$ para el B. No sabemos por qué lo hicieron así.

Los horóscopos ${ }^{72}$ no eran textos científicos, pero se elaboraron a partir de datos suministrados por los Diarios y Almanaques. Es difícil creer que hubo una casta de sacerdotes y adivinos, conocedores de estos tratados astronómicos y, sin

70 Los procedimientos son problemas en los que se indican los pasos a seguir para obtener la solución. La estructura de los mismos es similar a la encontrada en los ejercicios escolares de matemáticas; con algunas diferencias destacadas por ROBSON, E.: 2008, 226-227. Ver NEUGEBAUER, O.: ACT (1983).

71 O. NEUGEBAUER, O.: ACTI, 188 y ss.

72 ROCHBERG, F.: 2004. 
embargo, dedicados exclusivamente a preparar horóscopos y recitar presagios. ¿La configuración celestial influía sobre el destino de las personas o se trataba de un mensaje premonitorio enviado por los dioses? Una vez comprobada la periodicidad de los movimientos planetarios y la posibilidad de predecirlos, ¿hasta qué punto los astrónomos confiaban en el testimonio de las estrellas o, simplemente, buscaban satisfacer los requerimientos de una sociedad iletrada y constantemente amenazada?

El primer horóscopo escrito en cuneiforme data del año 410 a C. y el último se escribió en Babilonia en el año 69 de nuestra era. Solamente conservamos 28 documentos de esta clase. Todos estaban dedicados a personas individuales y en ellos se indican las posiciones de los planetas en relación al zodiaco en el día del nacimiento; situación de la Luna respecto a una estrella; pasados o futuros eclipses pronosticados para ese mismo año; fechas previstas para los solsticios y equinoccios. En ninguno de ellos se registran observaciones directas ni cálculos, pues se elaboran un tiempo después de haber tenido lugar el nacimiento. Proponemos leer dos horóscopos distintos; el primero está escrito por las dos caras de una misma tablilla y no advierte a quién va dirigido; el texto está dañado y es incompleto (los números indican las líneas) ${ }^{73}$ :

1. Año 92, [reinado de Antíoco (III).]

2. Tašritu (septiembre/octubre) 30, noche 1[2 (¿hora?), primera parte de la noche, la Luna estaba]

3. debajo de «la estrella posterior de la cabeza del Hombre» (se trata de $\alpha$ Aries).

4. La Luna pasó $1 / 2$ kùš al este (de $\alpha$ Aries)... [...]

5. el niño ha nacido; en [su] hora, [la Luna estaba en Aries ( $¿$ ?), ]

6. el Sol estaba en Escorpio, Júpiter [estaba en Aries],

7. Venus y Saturno (estaban) e[n Sagitario],

8. Mercurio y Marte [se habían puesto, no eran visibles.]

9. Ellos estaban junto al Sol. [Ese mes (i),]

1. El ocaso de la Luna, después de la salida del Sol, ocurrió a la (hora) 14 [última visibilidad lunar antes del orto del Sol el] (día) 2[7.]

2. [El solsticio de invierno sucedió] el (día) 20 de Kislīmu (noviembre/diciembre).

3. Addaru (febrero/marzo), noche del 1[4, un eclipse lunar,]

4. Todo (el eclipse) ocurr[ió] en Libra.

5. El (día) 28 un ecl[ipse de Sol]

6. en Aries, habiendo pasado medio mes (desde el eclipse anterior).

${ }^{73}$ ROCHBERG, F.: Ibid., 43-44. Las frases o palabras entre corchetes han sido reconstruidas por el editor de la tablilla. Las palabras entre paréntesis son aclaraciones. 
El segundo horóscopo es más breve y apenas contiene datos astronómicos; sin embargo, incluye pronósticos e indica el nombre del niño:

«Año 63 (era seleúcida), mes X, al atardecer del (día) 2, Anu-bēlšunu ha nacido. Ese día, el Sol estaba en 9;30ㅜ Capricornio, la Luna estaba en 12ำ Acuario: sus días serán largos. [Júpiter] estaba al comienzo de Escorpio: alguien ayudará al príncipe. [El niño] nació en Acuario en la región de Venus: tendrá hijos varones. Mercurio estaba en Capricornio; Saturno estaba en Capricornio; [Marte] en Cáncer. ${ }^{74}$

Estos documentos prueban la continuidad y vigencia de la tradición adivinatoria existente en Mesopotamia durante los períodos seleúcida y arsácida; tradición que alcanzó las civilizaciones de Grecia y Roma. Los escribas de Enūma Anu Enlil, dedicados a encontrar paralelismos entre los sucesos terrenales y los del cielo, acudían a consultar las Series cuando los monarcas y poderosos lo requerían; unas veces para tranquilizarles, otras, para prevenirles de iniciar determinada acción. Algunas familias encargaban un horóscopo para sus vástagos o acudían al adivino por cualquier otro motivo. Pues los habitantes de Mesopotamia seguían atemorizados por una naturaleza que solía actuar de manera violenta e impredecible.

\section{CONCLUSIÓN}

Entre las ciencias de la naturaleza, la astronomía es la más antigua de todas, pues nada se necesita para contemplar el firmamento e intentar descifrar el lenguaje de las estrellas. Los antiguos pobladores de Mesopotamia, creyeron que los dioses habitaban el cielo y que utilizaban los astros para comunicarse con los seres humanos. Posiblemente, algo tan fortuito e inesperado como la coincidencia entre la visión de un eclipse y la muerte de un monarca, pudo provocar que los sucesos naturales se entendieran como señales premonitorias acerca de fenómenos que podían afectar al estado y al reino. Saber interpretar la escritura celestial requería pericia e instrucción y solamente unos pocos escribas eran capaces de hacerlo. Aparentemente, las estrellas revolucionaban diariamente en torno a la Tierra y la Luna repetía sus fases. Había cierta rutina en estos movimientos que, sin embargo, no explicaba las trayectorias retrógradas de los planetas ni la aparición de eclipses y cometas. A fuerza de observar el cielo, intentando desvelar sus misterios, los astrónomos comprendieron que los cuerpos celestes renuevan sus fases periódicamente y que regresan al mismo lugar del que partieron decenas de años atrás. Los eclipses y otros fenómenos estelares, entonces, tenían una explicación natural que no dependía de las acciones humanas ni de la voluntad de los dioses; aunque estos podían seguir castigando y enviando toda clase de enfermedades y desventuras a aquellas personas que infringieran las normas ${ }^{75}$. La tradición adivinatoria, transmiti-

74 E. ROBSON comenta que sólo conservamos tres horóscopos nominativos (2008, 252-253).

75 Ver Traité akkadien de diagnostics et pronostics medicaux, R. LABAT, Leiden: Académie Internationale d'Histoire des Sciences (1951). Aquí, las enfermedades se conciben como el castigo que penaliza acciones contrarias a las normas. 
da de padres a hijos durante milenios, no desapareció porque se hubiera comprobado que los cielos rotan siguiendo pautas predecibles; pero abrió el camino hacia una nueva visión de la naturaleza que concibe el universo sujeto a leyes eternas e inmutables. No sabemos, por tanto, si los autores de los primeros textos científicos creían que la configuración estelar determina el futuro de las personas o, simplemente, se limitaban a desempeñar su oficio como habían hecho sus antecesores. Pues, lo que cuentan las estrellas, no siempre tiene que ver con nuestras cosas.

El conocimiento astronómico de los escribas babilonios se difundió a Oriente (India) y a Occidente (Grecia), en el período helenístico. Los árabes recogieron ambas tradiciones y las trasladaron a Europa en la Edad Media.

\section{BIBLIOGRAFÍA}

AABOE, A. (2002). «On Columns $\mathrm{H}$ and $\mathrm{J}$ in Babylonian lunar Theory of System B», en J. M. Steele \& A. Imhausen (2004) pp. 1-4.

AAVV (1966). La divination en Mésopotamie Ancienne et dans les régions voisines. Paris: PUF.

AAVV (1966). Le monde du sorcier. Paris: Éditions du Seuil.

BERGGREN, J. L. \& GOLDSTEIN, B. R. (1987). From Ancient Omens to Statistical Mechanics. Copenhagen: University Library.

BOTTÉRO, J. (1974). «Symptômes, signes, écritures en Mésopotamie Ancienne», en J. P. Vernant (1974), pp. 70-197.

BRACK-BERNSEN, L. \& H. HUNGER (1999). «The Babylonian Zodiac: Speculations on its invention and significance», Centaurus 41, pp. 280-292.

(2002). «Predictions of Lunar Phenomena in Babylonian Astronomy», en Steel \& Imhausen (2002).

CICERÓN, M. T. (1999). Sobre la adivinación. Madrid: Gredos.

GRASSHOFF, G. (1999). «Normal Star Observations in Late Babylonian Astronomical Diaries», en N. M. Swerdlow (1999), pp.97-147.

GRAY, J. M. K. \& STEELE, J. M. (2008). «Studies on Babylonian goal-year astronomy I: a comparison between planetary data in Goal-Year Texts, Almanacs and Normal Star Almanacs", Archive for History in Exact Sciences 62, pp. 553-600.

HIRSCH, H. (1963). Archiv für Orientforschung 20, Berlin.

HUBER, P. (1987). "Dating by Lunar Eclipse Omina, with Speculations on the Birth of Omen Astrology», en J. L. Berggren and B. R. Goldstein (1987), pp. 3-13.

HUNGER, H. \& PINGREE, D. (1999). Astral Sciences in Mesopotamia. Brill.

HUNGER, H. (1968). Babylonische und assyrische Kolophone, Alter Orient und Altes Testament 2 (Neukirchen-Vluyn: Verlag Butzon and Bercker Kevelaer), ํo 77.

(1999). «Non-mathematical Astronomical Texts and Their Relationships», en N. M. Swerdlow (1999), pp. 77-96).

KRAMER, S. N. (2010). La historia empieza en Sumer. Con Prólogo de F. Lara Peinado. Madrid: Alianza.

LARA PEINADO, F. (1982). Código de Hammurabi. Madrid: Editora Nacional.

(1987). Mitos sumerios y acadios. Madrid: Editora Nacional.

(1994). Enuma elish: poema babilónico de la creación. Madrid: Trotta.

LARSEN, M. T. (1987). «The Mesopotamian Lukewarm Mind, Reflections on Science, Divination and Literacy», en F. Rochberg-Halton (1987), pp. 203-225.

McEWAN, G. J. P. (1981). Priest and Temple in Hellenistic Babylonia. Wiesbaden: Franz Steiner. 
NEUGEBAUER, O. (1983). Astronomical Cuneiform Texts, 3 vols. Springer.

OPPENHEIM, A. L. (1966). Perspectives on Mesopotamian Divination, en AAVV, La divination en Mésopotamie Ancienne et dans les régions voisines, pp. 35-43.

(2003). La Antigua Mesopotamia. Gredos (Madrid).

PARPOLA, S. (1970). Letters from Assyrian Scholars to the Kings Esarhaddon and Assurbanipal, Alter Orient und Altes Testament (Parts I and II). Neukirchen-Vluyn: Verlag Butzon and Bercker Kevelaer.

REINER, E. (1999). «Babyonian Celestial Divination», en Swerdlow (2004).

REINER, E. \& PINGREE, D. (1978, 1981, 1998). Babylonian Planetary Omens, 3 Parts. Malibu, CA: Undena; and Groningen: Styx Publications.

ROBSON, E. (2008). Mathematics in Ancient Iraq. A Social History. Princeton University Press.

ROCHBERG-HALTON, F. (1987). Language, Literature, and History: Philological and Historical Studies Presented to Erica Reiner. New Haven: American Oriental Society.

ROCHBERG, F. (1999). «Babylonian Horoscopy: The Texts and Their Relations», en N. M. Swerdlow (1999), pp. 39-59.

(2004). The Heavenly Writing. Cambridge Univ. Press.

SACHS, A. J. (1948). «A Classification of the Babylonian Astronomical Tablets of the Seleucid Period», Journal of Cuneiform Studies 2, pp. 271-290.

STEELE, J. M.; A. IMHAUSEN (2002). Under One Sky. Münster: Ugarit Verlag.

SWERDLOW, N. M. (1999). Ancient Astronomy and Celestial Divination. MIT Press.

VERBRUGGHE, G. P.; WICKERSHAM, J. M. (1999). Berossos and Manetho. The University of Michigan Press.

VERNANT, J. P. et al. (1974). Divination et rationalité. Paris: Editions du Seuil. 
05-YUSTE 18/09/2012 14:27 Página 102 